\title{
Observational Bounds on Modified Gravity Models
}

\author{
Antonio De Felice ${ }^{1}$, Pia Mukherjee ${ }^{1}$, Yun Wang ${ }^{2}$ \\ ${ }^{1}$ Department of Physics \& Astronomy, \\ University of Sussex, Brighton BN1 9QH, United Kingdom. \\ ${ }^{2}$ Homer L. Dodge Department of Physics \& Astronomy, \\ University of Oklahoma, Norman, OK 73019, USA.
}

\begin{abstract}
Modified gravity provides a possible explanation for the currently observed cosmic accelaration. In this paper, we study general classes of modified gravity models. The Einstein-Hilbert action is modified by using general functions of the Ricci and the Gauss-Bonnet scalars, both in the metric and in the Palatini formalisms. We do not use an explicit form for the functions, but a general form with a valid Taylor expansion up to second order about redshift zero in the Riemann-scalars. The coefficients of this expansion are then reconstructed via the cosmic expansion history measured using current cosmological observations. These are the quantities of interest for theoretical considerations relating to ghosts and instabilities. We find that current data provide interesting constraints on the coefficients. The next-generation dark energy surveys should shrink the allowed parameter space for modifed gravity models quite dramatically.
\end{abstract}




\section{INTRODUCTION}

The discovery of the current accelerated expansion of the Universe [1, 2] has generated a lot of excitement in the last few years. It turns out that this unexpected behaviour can be modeled in many ways, most simply through a cosmological constant. On the other hand it has been argued that, since this behaviour appears on large scales, the acceleration of the universe is due to some modification of gravity on such scales.

There are quite a few models which can describe such modifications of gravity, such as scalar-tensor theories [3]-[15], the so called $f(R)$ theories [16]-[26] (which are a subset of scalar tensor theories), brane world models of which Randall-Sundrum models [27] and DGP models [28-31] are special cases, and some more complicated function of curvature invariants, such as $f\left(R, R_{\mathrm{GB}}^{2}\right)[32-36]$ where $R_{\mathrm{GB}}^{2}$ represents the Gauss-Bonnet combination defined as $R^{2}-4 R_{\alpha \beta} R^{\alpha \beta}+R_{\alpha \beta \gamma \delta} R^{\alpha \beta \gamma \delta}$.

Modifying gravity in a consistent way is not an easy task, hence many of these models face quite stringent theoretical bounds which can reduce the parameter space of these theories. In the same way one can try to use data to restrict further the parameter space and possibly rule out some of these models.

In this paper we study $f(R)$ and $f\left(R, R_{\mathrm{GB}}^{2}\right)$ models, most generally, without specifying any explicit form for these functions. In the $f(R)$ case, we consider both the metric based, and the Palatini formalisms. We use the recent expansion history of the universe, reconstructed allowing the Hubble parameter to be a free function in redshift bins, using type Ia supernovae (SNe Ia) data together with relevant contraints from the cosmic microwave background $(\mathrm{CMB})$ and galaxy surveys, and invert this to obtain constraints on the parameters of the $f(R)$ models. This perspective has not been adopted before. Although it is similar in nature to the reconstruction of the quintessence potential [37], here the scalar degrees of freedom come entirely from the gravity sector.

Much work has been dedicated to the subject of constraining $f(R)$ theories using solar system measurements [38]-[44]. We will not study this here for several reasons. First, as believed by some (e.g. [45]), the cosmological parameters may be different from those measured locally, because as the background changes from a locally spherically symmetric metric to a homogeneous and isotropic one, the behaviour of quantities such as the coefficients of a Taylor-expansion of $f(R)$ might be quite different. Second, for this theory locally (at the 
solar system scale) the weak field approximation may not hold, so that it cannot be matched to a perturbation of GR; i.e., the usual constraints cannot be trivially applied because these are found by assuming the metric to be a perturbation about the GR-Schwarzschild solution [46].

Furthermore we are restricting our study to the background evolution at low redshifts, assuming GR-like behaviour until then. We are not taking into account perturbations and their evolution, nor considering the stability of modes in the early universe, in constraining the models. These relevant issues have been studied in [18, 23, 25, 35, 36, 47-49].

This paper is organized as follows. In Section II we discuss the general metric based $f(R)$ case and its solutions. In Section III we discuss the Palatini formalism and its solutions. In Section IV we consider the generalization including the Gauss-Bonnet term. We end with a discussion and conclusions section. Appendices follow.

\section{GENERAL METRIC-BASED $f(R)$ MODEL}

Let us begin with an $f(R)$ theory with the following action

$$
S=\int d^{4} x \sqrt{-g} \frac{R+f(R)}{16 \pi G}+S_{m}
$$

where $R$ is the Ricci scalar and $S_{m}$ is the action for the matter fields. The equations of motion are

$$
\left(1+f_{R}\right) G_{\mu \nu}-\frac{1}{2} g_{\mu \nu}\left(f-R f_{R}\right)+g_{\mu \nu} \square f_{R}-\nabla_{\mu} \nabla_{\nu} f_{R}=8 \pi G T_{\mu \nu}
$$

where an underscore $R$ implies a partial derivative with respect to $R\left(f_{R}=\partial f / \partial R\right)$. In a FRW background then,

$$
3\left(1+f_{R}\right) H^{2}+\frac{1}{2}\left(f-R f_{R}\right)+3 H^{2} f_{R}^{\prime}=8 \pi G \rho,
$$

where $H$ is the Hubble parameter and a prime denotes differentiation with respect to $N=$ $\ln \left(a / a_{0}\right)$. Evaluated today this equation becomes

$$
1+\beta+\frac{1}{6}\left[\alpha-6 \beta\left(H_{0}^{\prime} / H_{0}+2\right)\right]+\gamma \frac{R_{0}^{\prime}}{H_{0}^{2}}=\frac{8 \pi G}{3 H_{0}^{2}} \rho_{0},
$$

where an underscore 0 implies present values, and $\alpha=f_{0} / H_{0}^{2}, \beta=f_{R 0}$, and $\gamma=f_{R R 0} H_{0}^{2}$ are the dimension-less parameters of this theory. Since we have three parameters to solve 
for, we need three equations. These are obtained by differentiating the Friedmann equation twice. The equations then involve higher derivatives of $H$ evaluated today (in this case upto four).

Assuming that $f$ can be Taylor-expanded in $R$ about today and retaining only up to the second order terms (this being the simplest non-trivial case),

$$
f \approx f_{0}+f_{R 0} H_{0}^{2}\left[\frac{R-R_{0}}{H_{0}^{2}}\right]+\frac{1}{2} f_{R R 0} H_{0}^{4}\left[\frac{R-R_{0}}{H_{0}^{2}}\right]^{2} .
$$

In order to use the equations of motion effectively one needs to truncate the Taylor expansion at some order, otherwise one would need an infinite number of equations, obtained from repeatedly differentiating the Friedmann equation, to specify an infinite number of parameters, the Taylor coefficients. We chose to truncate this expansion of $f(R)$ at second order because stability constraints on these theories involve $f_{R}$ and $f_{R R}$ terms (see e.g. [23]).

Differentiating the Friedmann equation once gives

$$
6 H H^{\prime}\left(1+f_{R}\right)+3\left(H H^{\prime}-H^{2}\right) f_{R}^{\prime}+3 H^{2} f_{R}^{\prime \prime}=8 \pi G \rho^{\prime} .
$$

This relation together with the conservation of stress-energy (which ignoring contribution from radiation leads to)

$$
\rho^{\prime}=-3(1+w) \rho \approx-3 \rho
$$

is equivalent to the second Einstein equation. Equations (4), (5) and (6) lead to

$$
6 \mathcal{H}^{\prime}\left(1+f_{R 0}\right)+3\left(\mathcal{H}^{\prime}-1\right) f_{R R 0} R_{0}^{\prime}+3 f_{R R 0} R_{0}^{\prime \prime}=-9 \Omega_{m}
$$

where we have defined the (present day) derivatives of the (normalized) Hubble parameter as

$$
\mathcal{H}^{\prime}=\frac{H_{0}^{\prime}}{H_{0}} \quad \text { and } \quad \mathcal{H}^{\prime \prime}=\frac{H_{0}^{\prime \prime}}{H_{0}} .
$$

For a flat Friedmann-Robertson-Walker background

$$
\begin{aligned}
R & =6\left(H H^{\prime}+2 H^{2}\right) \\
R^{\prime} & =6\left(H^{\prime 2}+H H^{\prime \prime}+4 H H^{\prime}\right) \\
R^{\prime \prime} & =6\left(H H^{\prime \prime \prime}+3 H^{\prime} H^{\prime \prime}+4 H^{2}+4 H H^{\prime \prime}\right)
\end{aligned}
$$

hence equation (7) can also be written as

$$
\beta=-\frac{2 \mathcal{H}^{\prime}+3 \Omega_{m}+6 \gamma\left(\mathcal{H}^{\prime \prime \prime}+3 \mathcal{H}^{\prime \prime}+4 \mathcal{H}^{\prime} \mathcal{H}^{\prime \prime}+\mathcal{H}^{\prime 3}+7 \mathcal{H}^{\prime 2}-4 \mathcal{H}^{\prime}\right)}{2 \mathcal{H}^{\prime}}
$$


Differentiating the Friedmann equation a second time, evaluating it today and using equation (10) gives

$$
\begin{array}{r}
\gamma=\frac{1}{2} \Omega_{m}\left(3 \mathcal{H}^{\prime}+\mathcal{H}^{\prime 2}+\mathcal{H}^{\prime \prime}\right) \times\left[9 \mathcal{H}^{\prime 4}+15 \mathcal{H}^{2} \mathcal{H}^{\prime \prime}+6 \mathcal{H}^{\prime 3} \mathcal{H}^{\prime \prime}\right. \\
\left.-3 \mathcal{H}^{\prime \prime 2}+3 \mathcal{H}^{\prime} \mathcal{H}^{\prime \prime \prime \prime}+6 \mathcal{H}^{\prime 2} \mathcal{H}^{\prime \prime \prime}-\mathcal{H}^{\prime \prime} \mathcal{H}^{\prime \prime \prime}+\mathcal{H}^{\prime} \mathcal{H}^{\prime \prime \prime \prime}\right]^{-1}
\end{array}
$$

where

$$
\mathcal{H}^{\prime \prime \prime} \equiv \frac{H_{0}^{\prime \prime \prime}}{H_{0}} \quad \text { and } \quad \mathcal{H}^{\prime \prime \prime \prime} \equiv \frac{H_{0}^{\prime \prime \prime \prime}}{H_{0}}
$$

We see that

$$
\alpha=\alpha\left(\mathcal{H}^{\prime}, \mathcal{H}^{\prime \prime}, \mathcal{H}^{\prime \prime \prime}, \mathcal{H}^{\prime \prime \prime \prime}, \Omega_{m}\right)
$$

and the same is true for $\beta$ and $\gamma$. The relations are non-linear.

Posterior distributions of $\mathcal{H}^{\prime}, \mathcal{H}^{\prime \prime}, \mathcal{H}^{\prime \prime \prime}, \mathcal{H}^{\prime \prime \prime \prime}$ and $\Omega_{m}$ are obtained from a likelihood analysis of data in the following way. We use 182 Type Ia supernovae (SNe Ia) from the HST/GOODS program, together with first year SNLS and some nearby SNe Ia, as compiled by [50]. The $\left(R, l_{a}, \Omega_{b} h^{2}\right)$ combination, where $R$ and $l_{a}$ are CMB shift parameters[51], is used to account for relevant constraints from the CMB [52]. The SDSS baryon acoustic oscillation (BAO) scale measurement is also used [53]. Following the analysis method of [54]-[56],[51], model independent constraints are derived on the Hubble parameter in linear redshift bins using a Markov Chain Monte Carlo (MCMC) algorithm. The $H(z)$ 's of the MCMC chain elements are then converted into the derivatives of $\mathcal{H}$, as described in Appendix A. The top panel of Fig 1 shows the constraints thus derived on what we for convenience shall sometimes refer to as the "initial parameters". From the MCMC chains now we can obtain constraints on $\alpha, \beta$, and $\gamma$ using the equations derived above.

\section{Linearization}

Equation (11) is highly non-linear in the initial parameters. Small changes in these parameters can have a large effect on the values of the $f(R)$ parameters, so that a relatively small number of high likelihood combinations of the initial parameters can lead to a large range of values for the $f(R)$ parameters. This leads to flat 1D parameter likelihood curves. This effect is suppressed in the MCMC posterior of the $f(R)$ parameters, because in addition to the likelihood this takes into account the number of samples that fall into each bin. In 

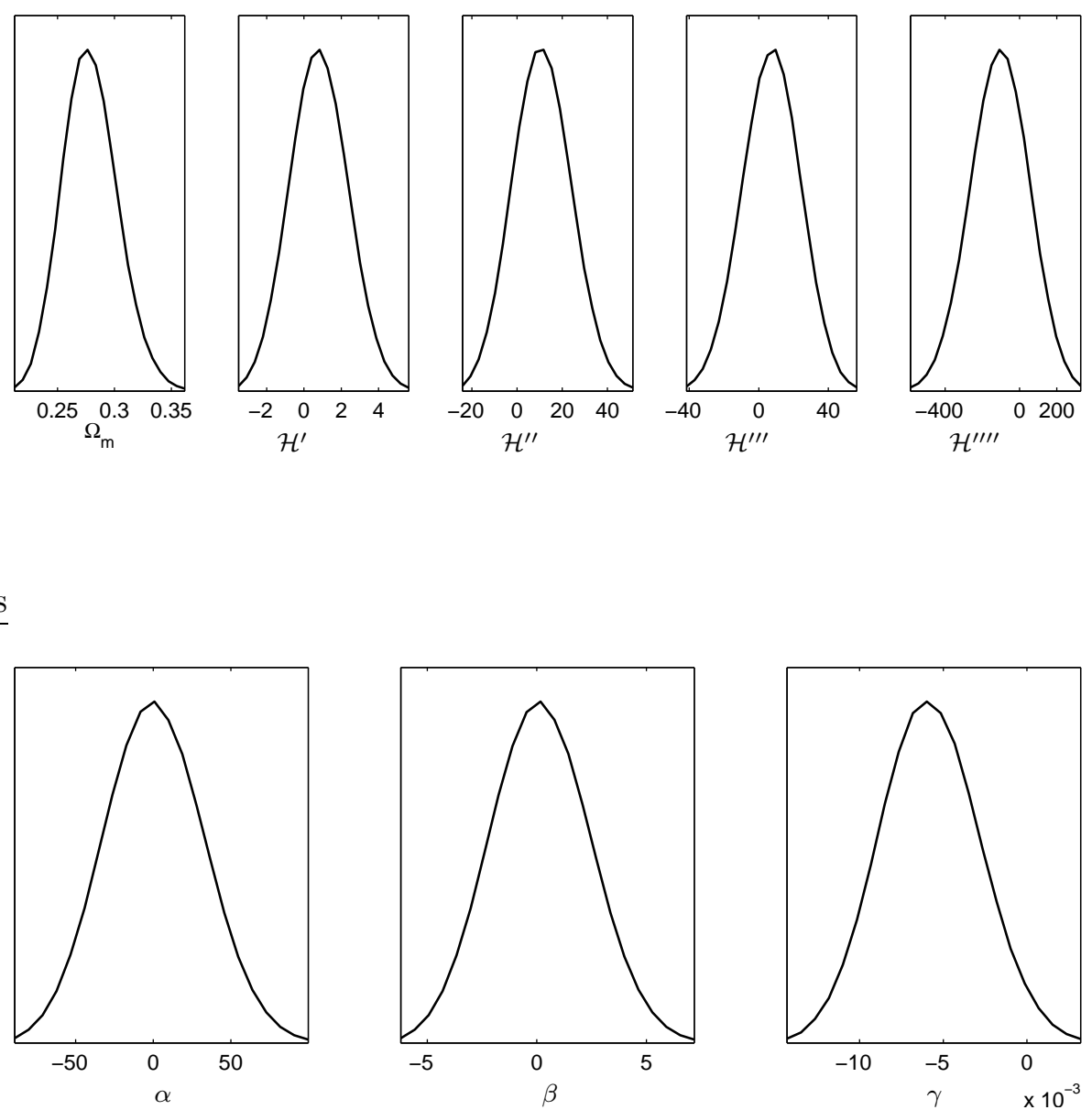

FIG. 1: Results for general metric-based $f(R)$ gravity: constraints on $\alpha=f_{0} / H_{0}^{2}, \beta=f_{R 0}$, and $\gamma=f_{R R 0} H_{0}^{2}$ (bottom panel), obtained from constraints on $\Omega_{m}$ and the derivatives of $\mathcal{H}$ (top panel), using current cosmological data.

other words the discrepancy between these curves is due to there being a main posterior peak (representing a concentration of high likelihood points) together with isolated points in parameter space that are allowed by the likelihood. In addition, some allowed values of the initial parameters lead to singularities; these are the zero values of the $\mathcal{H}^{\prime \prime}$ s and the zeroes of denominator in the expression for $\gamma$. Given the discrete samples in the MCMC chain, the parameters $\alpha, \beta$ and $\gamma$ don't actually blow up, but instead as a result have a larger range. These problems should be at least somewhat eased by better data. For now, in order to avoid the discrepancy between the likelihood and the posterior, and to discount the region around singular points, we proceed to find solutions by first linearizing the equations for the modified gravity parameters about the mean values of the initial parameters, supported also 


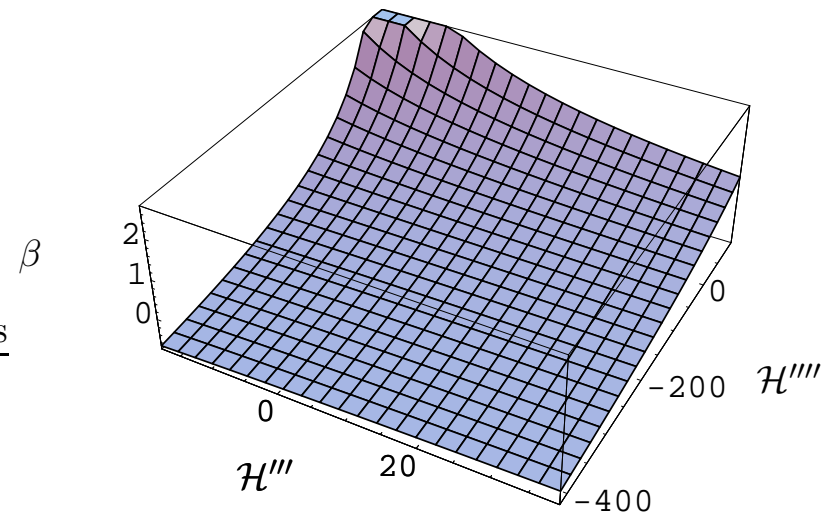

FIG. 2: $\beta$ as a function of $\mathcal{H}^{\prime \prime \prime}$ and $\mathcal{H}^{\prime \prime \prime \prime \prime}$ setting $\mathcal{H}^{\prime}$ and $\mathcal{H}^{\prime \prime}$ to their best values.

by the fact that the initial parameters have close to Gaussian distributions. This approach will be used in subsequent sections as well.

We linearize the equations for $\alpha, \beta$ and $\gamma$ about their maximum likelihood (in MCMC, the relevant quantity is the mean) values, which are obtained in turn from the mean values of the initial parameters:

$$
\begin{aligned}
& \alpha \approx \hat{\alpha}+\left.\left(p_{i}-\hat{p}_{i}\right) \frac{\partial \alpha}{\partial p_{i}}\right|_{p_{j}=\hat{p}_{j}}, \\
& \beta \approx \hat{\beta}+\left.\left(p_{i}-\hat{p}_{i}\right) \frac{\partial \beta}{\partial p_{i}}\right|_{p_{j}=\hat{p}_{j}}, \\
& \gamma \approx \hat{\gamma}+\left.\left(p_{i}-\hat{p}_{i}\right) \frac{\partial \gamma}{\partial p_{i}}\right|_{p_{j}=\hat{p}_{j}},
\end{aligned}
$$

where $p_{i}=\left(\mathcal{H}^{\prime}, \mathcal{H}^{\prime \prime}, \mathcal{H}^{\prime \prime \prime}, \mathcal{H}^{\prime \prime \prime \prime}, \Omega_{m}\right)$ and hats represent mean values.

We obtain

$$
\begin{aligned}
\alpha=- & 29.7708+31.9923 \mathcal{H}^{\prime}-0.652908 \mathcal{H}^{\prime \prime}-0.0628292 \mathcal{H}^{\prime \prime \prime} \\
& +0.0513295 \mathcal{H}^{\prime \prime \prime \prime}+63.1444 \Omega_{m}, \\
\beta=- & 1.89148+2.29135 \mathcal{H}^{\prime}-0.0479787 \mathcal{H}^{\prime \prime}-0.00179758 \mathcal{H}^{\prime \prime \prime} \\
& +0.00402273 \mathcal{H}^{\prime \prime \prime \prime}+4.20931 \Omega_{m}, \\
\gamma=- & 0.00179067-0.00887807 \mathcal{H}^{\prime}+0.000625996 \mathcal{H}^{\prime \prime}+0.0000797733 \mathcal{H}^{\prime \prime \prime} \\
& -0.0000138531 \mathcal{H}^{\prime \prime \prime \prime}-0.02083 \Omega_{m} .
\end{aligned}
$$


Even though the linearization is about the mean values of the initial parameters, we then use the MCMC chain to obtain the distributions of the modified gravity parameters. These are shown in the bottom panel of Fig 1. As described above these represent the main body of solutions of the $f(R)$ theory allowed by the data; given the non-linearity of the equations there are other solutions isolated in parameter space. We see that while the order of magnitude of $\alpha$ and $\beta$ is constrained, $\gamma \propto f_{R R, 0}$ is found to be slightly negative today over most of its allowed range. Even though we are not discussing stability issues here, $[20,23,24,26]$ find that $f_{R R}$ at high redshift needs to be positive in order to avoid instability and obey GR. Therefore under this theory $\gamma$ would have to change sign at some $R$ before today. This is interesting.

Fig 2 shows the solutions for $\beta$ at the mean values of the better constrained derivatives $\mathcal{H}^{\prime}$ and $\mathcal{H}^{\prime \prime}$, and over the allowed ranges of the higher derivatives. A range of values for $\beta$ are possible, including the special case of $\beta=1 / 3$ discussed below.

\section{A. Metric based $f(R)$ with $\beta=1 / 3$}

If we assume that in the solar system the real metric can be expanded about GR Schwarzchild, then for the previous action, the effective Newton's constant can be written as $[12]$

$$
G_{\mathrm{eff}}=\frac{4}{3} \frac{G}{1+f_{R}}
$$

which can be recast as a constraint on $\beta$ today (imposing $G=G_{\text {eff }, 0}$ )

$$
f_{R 0}=\beta=\frac{1}{3} \text {. }
$$

Imposing $\beta=1 / 3$, equation (3) and (10) give

$$
\frac{1}{6} \alpha-\frac{1}{3}\left(\mathcal{H}^{\prime}+2\right)+6 \gamma\left(\mathcal{H}^{\prime \prime}+\mathcal{H}^{\prime 2}+4 \mathcal{H}^{\prime}\right)=\Omega_{m}-\frac{4}{3},
$$

and

$$
\frac{8}{3} \mathcal{H}^{\prime}+6 \gamma\left[3 \mathcal{H}^{\prime \prime}+\mathcal{H}^{\prime}\left(\mathcal{H}^{\prime 2}+7 \mathcal{H}^{\prime}+4 \mathcal{H}^{\prime \prime}-4\right)+\mathcal{H}^{\prime \prime \prime}\right]=-3 \Omega_{m}
$$

The data define constraints on the $\mathcal{H}^{\prime}$ 's as discussed in the previous section. Equations (21) and (22) can then be used to solve for $\alpha$ and $\gamma$. The complications arising due to the equations being non-linear in the initial parameters, as discussed earlier, apply here as well. Hence as before we proceed to linearize the equations of motion about the mean values of the $\mathcal{H}^{\prime \prime}$ s and $\Omega_{m}$. 


\section{Linearization}

Using equations (14) and (16) together with equations (21) and (22), we get

$$
\begin{aligned}
& \alpha \approx-4.53219+12.1526 \mathcal{H}^{\prime}-1.54509 \mathcal{H}^{\prime \prime}-0.974158 \mathcal{H}^{\prime \prime \prime}+20.5967 \Omega_{m} \\
& \gamma \approx-0.028308-0.0521268 \mathcal{H}^{\prime}+0.0262386 \mathcal{H}^{\prime \prime}+0.00930701 \mathcal{H}^{\prime \prime \prime}-0.139456 \Omega_{m}
\end{aligned}
$$

These relations, used on the MCMC chains, give the results shown in Fig 3.
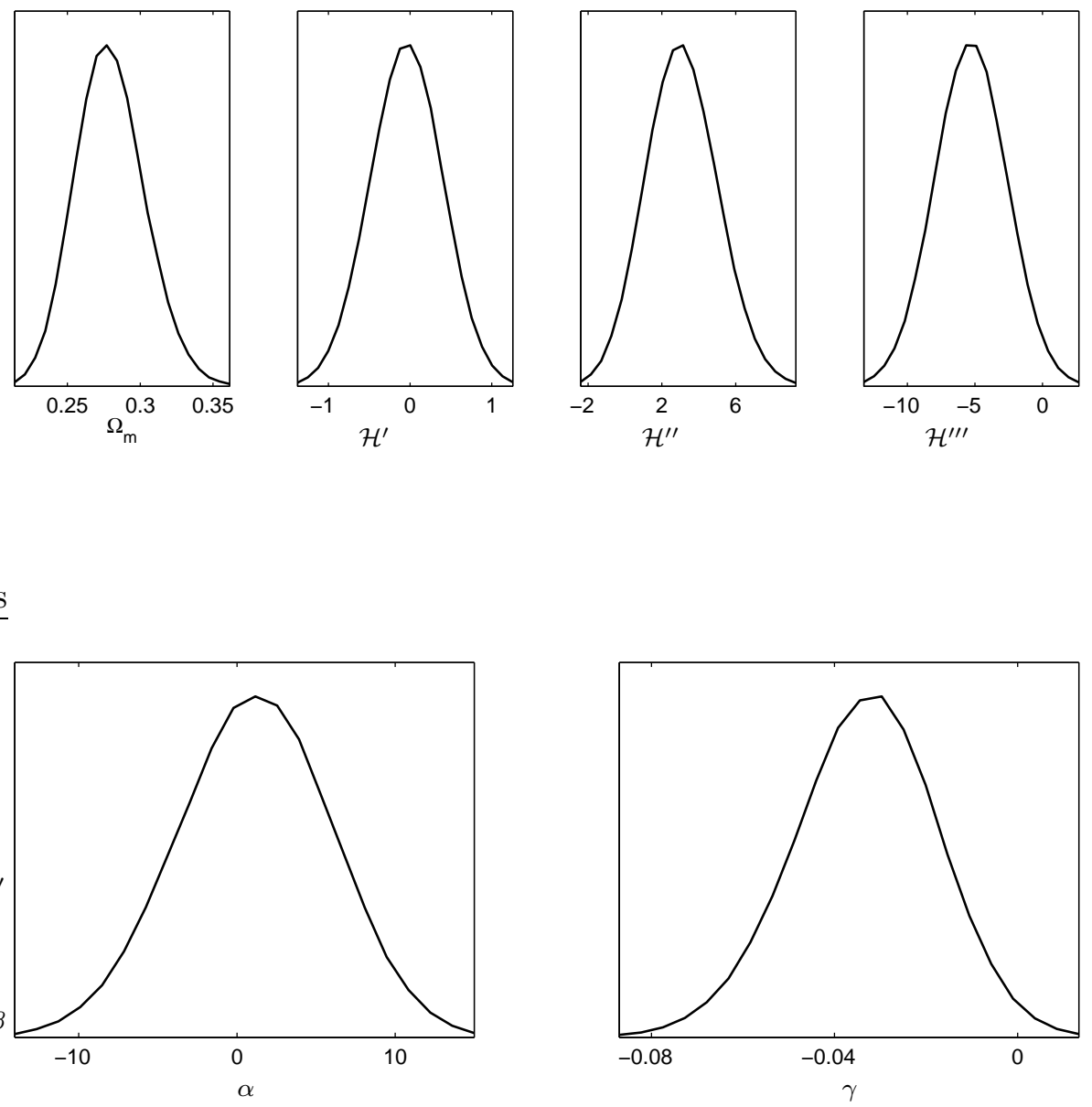

FIG. 3: Same as Fig 1 for the $\beta=\frac{1}{3}$ case of the general metric-based $f(R)$ theories.

\section{III. $f(R)$ IN THE PALATINI FORMALISM}

So far we have considered theories for which the gravity variables were chosen to be the metric elements $g_{\mu \nu}$. On the other hand, after writing down the action, one is free to 
choose a different set of fields. For example, one can choose to find the equations of motion by varying the action with respect to the following two tensorial quantities, $\delta g_{\mu \nu}$ and $\delta \Gamma_{\mu \nu}^{\lambda}$, which are the metric and the Christoffel symbols perturbations respectively. In standard GR, the approach of choosing two different field variables, leads to the same standard Einstein equations of motion. However, in $f(R)$ theories, with $f_{R} \neq$ constant, the equations of motion are indeed different. Of course, one may wonder which description of gravity is the correct one. However, since gravity is the least well known force, many physicists have argued that the possibility of introducing extra fields should be considered.

In this formalism (refered to as Palatini, also studied in [19, 57-59]), $R_{\mu \nu}$ becomes a function only of the Christoffel symbols, so that it cannot be written as usual in terms of derivatives of the scale factor. In this case it is customary to write the general action in the following way

$$
S=\int d^{4} x \sqrt{-g} \frac{f(R)}{16 \pi G}+S_{m} .
$$

The Einstein equations obtained by varying the action with respect to the metric elements are

$$
f_{R} R_{\mu \nu}-\frac{1}{2} f g_{\mu \nu}=8 \pi G T_{\mu \nu}
$$

the trace of which is

$$
f_{R} R-2 f=-8 \pi G \rho_{m}
$$

Taylor-expanding $f$ in terms of $R$ about today, as before, we have

$$
\bar{f} \equiv \frac{f}{H_{0}^{2}}=\alpha+\beta\left(\bar{R}-\bar{R}_{0}\right)+\frac{1}{2} \gamma\left(\bar{R}-\bar{R}_{0}\right)^{2}
$$

where $\bar{R} \equiv R / H_{0}^{2}$, and $\bar{R}_{0}$ is its value today. Therefore from equation (27)

$$
\bar{R}_{0}=\frac{2 \alpha-3 \Omega_{m}}{\beta}
$$

and in general

$$
\bar{R} \equiv \frac{R}{H_{0}^{2}}=\frac{2 \alpha-2 \beta \bar{R}_{0}+\gamma \bar{R}_{0}^{2}-3 \Omega_{m} e^{-3 N}}{\gamma \bar{R}_{0}-\beta},
$$

This relation is important as we know how $R$ varies close to today. Taking the derivative of $R$ with repect to time, plugging it into the 00 component of equation (26), we get the new Friedmann equation

$$
\frac{H^{2}}{H_{0}^{2}}=\frac{6 \Omega_{m} e^{-3 N}+f_{R} \bar{R}-\bar{f}}{6 f_{R} \xi}
$$


with $H=H(N)$ is the Hubble factor $H=a^{-1} d a / d t$, and

$$
\xi=\frac{\left[2 e^{3 N}\left(\beta^{2}-2 \alpha \gamma\right)-3 \gamma \Omega_{m}\right]^{2}}{4\left[e^{3 N}\left(\beta^{2}-2 \alpha \gamma\right)+3 \gamma \Omega_{m}\right]^{2}}
$$

following [19].

The Friedmann equation (31) together with its two $N$-derivatives give us three equations for three unknowns. In contrast to the metric-based case, the Friedmann equation here does not involve any derivatives of the Hubble parameter, because $R$ is independent of $H$. We end up with three non-linear equations involving $\Omega_{m}, \mathcal{H}^{\prime}, \mathcal{H}^{\prime \prime}$, to be solved simultaneously for $\alpha, \beta$ and $\gamma$. This time though, the equations are non-linear even in $\alpha, \beta$, and $\gamma$, so that there isn't a unique solution for these parameters. In general there is no telling how many real solutions there are. We solve the equations numerically in the range $[-10,10]$ for the parameters. Listed in Appendix B are multiple solutions found at the mean values of the initial parameters. Next we consider one of these solutions.

\section{Linearization}

The equations of motion can be written as $F_{i}\left(p_{j}, q_{k}\right)=0$, with $i=1,2,3$, where $F_{1}=0$ corresponds to the Friedmann equation, and $F_{2}=0$, and $F_{3}=0$ to its two derivatives. Let $p_{j}=(\alpha, \beta, \gamma)$ and $q_{k}=\left(\Omega_{m}, \mathcal{H}^{\prime}, \mathcal{H}^{\prime \prime}\right)$. The solutions listed in Appendix B are the $\hat{p}_{j}$ which solve $F_{i}\left(\hat{p}_{j}, \hat{q}_{k}\right)=0$, where $\hat{q}_{k}$ correspond to the mean values of the initial parameters. About any one solution we may linearize the equations of motion (for reasons explained in section II):

$$
F_{i}\left(p_{j}, q_{k}\right)=0 \approx F_{i}\left(\hat{p}_{j}, \hat{q}_{k}\right)+\left.\frac{\partial F_{i}}{\partial p_{j}}\right|_{\hat{p}, \hat{q}}\left(p_{j}-\hat{p}_{j}\right)+\left.\frac{\partial F_{i}}{\partial q_{j}}\right|_{\hat{p}, \hat{q}}\left(q_{j}-\hat{q}_{j}\right) .
$$

With $A_{i j}=\partial F_{i} /\left.\partial p_{j}\right|_{\hat{p}, \hat{q}}$ and $B_{i k}=\partial F_{i} /\left.\partial q_{k}\right|_{\hat{p}, \hat{q}}$, one has

$$
p_{j}=-\left(A^{-1} B\right)_{j k} q_{k}+\hat{p}_{j}+\left(A^{-1} B\right)_{j k} \hat{q}_{k} .
$$

As an example, consider the solution [0.222102, 0.00488155, 4.12207e-05]. We can obtain the distributions of $p_{j}$ about this solution using the linearized equations together on the MCMC chains for the initial parameters. Results are shown in Fig 4, and for this solution the matrix $A^{-1} B$ is also given in Appendix B. Though the figure shows reasonably strong constraints on the modified gravity parameters, it should be remembered that in this formalism the data allow multiple such solutions. One may try to distinguish between 
the solutions by imposing consistency checks derived from taking higher derivatives of the Friedmann equation. In that case we would use constraints on the Hubble parameter in more redshift bins, which would allow more freedom and hence more solutions overall to distinguish between (even though here we are only discussing solutions about one set of values for the initial parameters). Hence this endevour will not be fruitful at this time. With much better data, the solutions may be tractable.
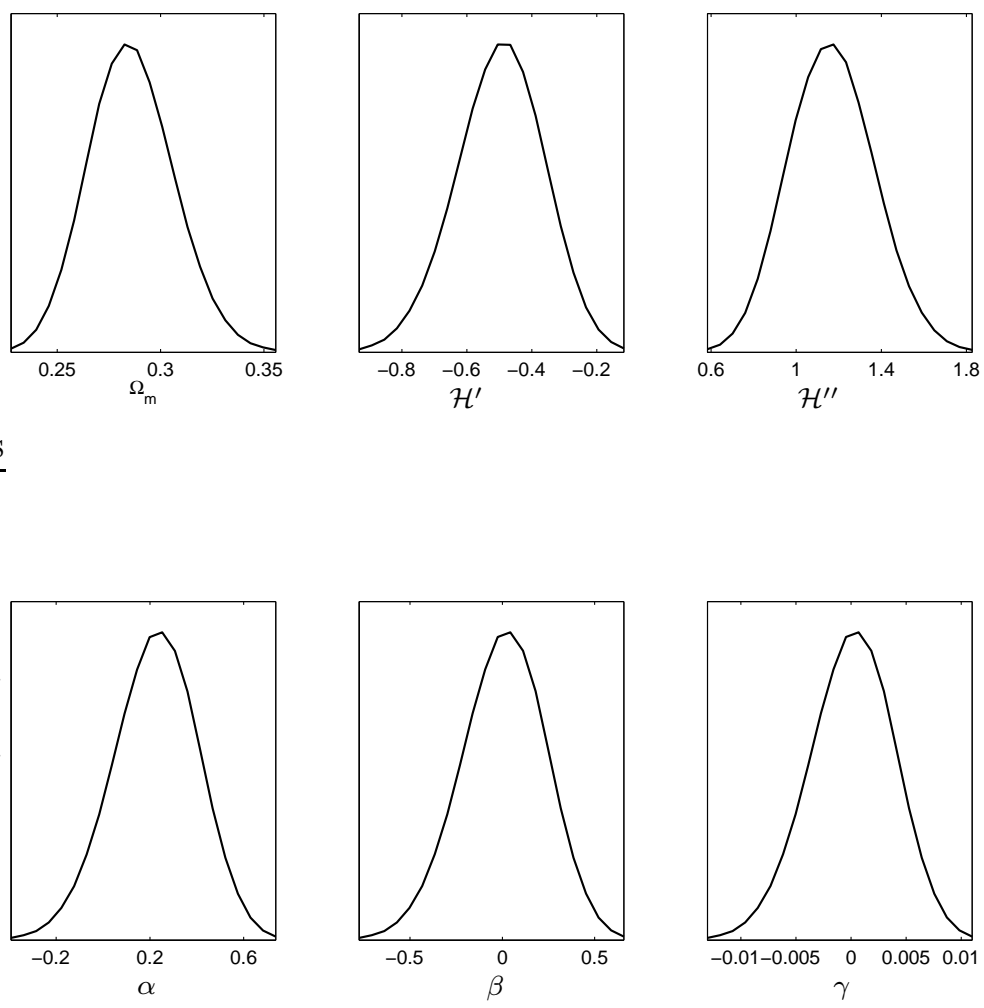

FIG. 4: Same as Fig 1, for $f(R)$ gravity in the Palatini formalism. The constraints in the bottom panel are about one solution of the three non-linear equations of motion. There are other solutions, see text for further discussion.

We also note in passing that when we constrain just two $\mathcal{H}^{\prime}$ 's, these turn out to be constrained to non-zero values (Fig 4). A negative $\mathcal{H}^{\prime}$ implies that the universe is not superaccelerating today, and positive $\mathcal{H}^{\prime \prime}$ implies that $\mathcal{H}^{\prime}$ is increasing (approaching zero) today. This is as expected. When more $\mathcal{H}^{\prime}$ 's are involved, as in the remaining sections, they of course become less constrained. 


\section{IV. $f\left(R, R_{\mathrm{GB}}^{2}\right)$ METRIC-BASED THEORIES}

It would be interesting to look also at other generalizations which have been considered in the literature [32]-[35]. These models have actions of the form

$$
S=\frac{1}{16 \pi G} \int d^{4} x \sqrt{-g}\left[R+f\left(R, R_{\mathrm{GB}}^{2}\right)\right]+S_{m},
$$

where $R_{\mathrm{GB}}^{2}=R^{2}-4 R_{\mu \nu} R^{\mu \nu}+R_{\mu \nu \rho \sigma} R^{\mu \nu \rho \sigma}$ is the Gauss-Bonnet combination. The equations of motion which follow can be written as

$$
\begin{aligned}
& (1+F) G_{\alpha \beta}-\nabla_{\alpha} \nabla_{\beta} F+g_{\alpha \beta} \square F-\frac{1}{2} g_{\alpha \beta}\left(f-F R-\xi R_{\mathrm{GB}}^{2}\right) \\
& \quad-2 R \nabla_{\alpha} \nabla_{\beta} \xi+2 R g_{\alpha \beta} \square \xi-4 R_{\alpha \beta} \square \xi-4 R_{(\alpha}{ }^{\sigma \tau}{ }_{\beta)} \nabla_{\sigma} \nabla_{\tau} \xi \\
& \quad-4 g_{\alpha \beta} R^{\rho \sigma} \nabla_{\rho} \nabla_{\sigma} \xi+8 R_{(\alpha}{ }^{\nu} \nabla_{\beta)} \nabla_{\nu} \xi=8 \pi G T_{\alpha \beta},
\end{aligned}
$$

where $F=\partial f / \partial R$ and $\xi=\partial f / \partial R_{\mathrm{GB}}^{2}$ and the partial derivatives should be found treating $R$ and $R_{\mathrm{GB}}^{2}$ as independent variables. In a FRW background this becomes

$$
3 H^{2}\left(1+F+F^{\prime}\right)+\frac{1}{2}\left(f-F R-\xi R_{\mathrm{GB}}^{2}\right)+12 H^{4} \xi^{\prime}=8 \pi G \rho,
$$

where

$$
R_{\mathrm{GB}}^{2}=24 \frac{\ddot{a}}{a} H^{2}=24 H^{3}\left(H^{\prime}+H\right),
$$

and $R$ has been already introduced in equations (9).

The Taylor-expansion of $f$ about today up to second order in the scalars takes the form

$$
\begin{aligned}
\frac{f}{H_{0}^{2}}=\alpha & +\beta\left(\bar{R}-\bar{R}_{0}\right)+\frac{1}{2} \gamma_{1}\left(\bar{R}-\bar{R}_{0}\right)^{2} \\
& +\gamma_{2}\left(\bar{R}-\bar{R}_{0}\right)\left(\bar{R}_{\mathrm{GB}}^{2}-\bar{R}_{\mathrm{GB} 0}^{2}\right)+\frac{1}{2} \gamma_{3}\left(\bar{R}_{\mathrm{GB}}^{2}-\bar{R}_{\mathrm{GB} 0}^{2}\right)^{2},
\end{aligned}
$$

where

$$
\alpha \equiv \frac{f_{0}}{H_{0}^{2}}, \beta \equiv F_{0},\left.\gamma_{1} \equiv H_{0}^{2} \frac{\partial F}{\partial R}\right|_{0},\left.\gamma_{2} \equiv H_{0}^{4} \frac{\partial \xi}{\partial R}\right|_{0},\left.\gamma_{3} \equiv H_{0}^{6} \frac{\partial \xi}{\partial R_{\mathrm{GB}}^{2}}\right|_{0},
$$

with $\bar{R}=R / H_{0}^{2}$ and $\bar{R}_{\mathrm{GB}}^{2}=R_{\mathrm{GB}}^{2} / H_{0}^{4}$. It should be noted that there is no linear term in the expansion for $R_{\mathrm{GB}}^{2}$, because such a term would give no contribution to the equations of motion, and the constant term in $\left.\bar{R}_{\mathrm{GB}}^{2}\right|_{0}$ is considered absorbed in $\alpha$. Then one has

$$
\begin{aligned}
F & =\beta+\gamma_{1}\left(\bar{R}-\bar{R}_{0}\right)+\gamma_{2}\left(\bar{R}_{\mathrm{GB}}^{2}-\bar{R}_{\mathrm{GB} 0}^{2}\right), \\
H_{0}^{2} \xi & =\gamma_{2}\left(\bar{R}-\bar{R}_{0}\right)+\gamma_{3}\left(\bar{R}_{\mathrm{GB}}^{2}-\bar{R}_{\mathrm{GB} 0}^{2}\right) .
\end{aligned}
$$


Today equation (37) becomes

$$
\begin{aligned}
\alpha=6[ & -1+\left(1+\mathcal{H}^{\prime}\right) \beta-6 \gamma_{1}\left(4 \mathcal{H}^{\prime}+\mathcal{H}^{\prime 2}+\mathcal{H}^{\prime \prime}\right) \\
& -48 \gamma_{2}\left(4 \mathcal{H}^{\prime}+2 \mathcal{H}^{\prime 2}+\mathcal{H}^{\prime \prime}\right) \\
& \left.-96 \gamma_{3}\left(4 \mathcal{H}^{\prime}+3 \mathcal{H}^{\prime 2}+\mathcal{H}^{\prime \prime}\right)+\Omega_{m}\right]
\end{aligned}
$$

Taking four derivatives of equation (37) allows us to solve for the five parameters in term of $\Omega_{m}$ and the the derivatives of $\mathcal{H}$ (up to the 6th derivative). One derivative gives

$$
\begin{aligned}
\beta=-1 & +\left(2 \mathcal{H}^{\prime}\right)^{-1}\left[6\left(4 \mathcal{H}^{\prime}-7 \mathcal{H}^{\prime 2}-\mathcal{H}^{\prime 3}-3 \mathcal{H}^{\prime \prime}-4 \mathcal{H}^{\prime} \mathcal{H}^{\prime \prime}-\mathcal{H}^{\prime \prime \prime}\right) \gamma_{1}\right] \\
& +\left(2 \mathcal{H}^{\prime}\right)^{-1}\left[48\left(4 \mathcal{H}^{\prime}-14 \mathcal{H}^{\prime 2}-6 \mathcal{H}^{\prime 3}-3 \mathcal{H}^{\prime \prime}-8 \mathcal{H}^{\prime} \mathcal{H}^{\prime \prime}-\mathcal{H}^{\prime \prime \prime}\right) \gamma_{2}\right] \\
& +\left(2 \mathcal{H}^{\prime}\right)^{-1}\left[96\left(4 \mathcal{H}^{\prime}-21 \mathcal{H}^{\prime 2}-15 \mathcal{H}^{\prime 3}-3 \mathcal{H}^{\prime \prime}-12 \mathcal{H}^{\prime} \mathcal{H}^{\prime \prime}-\mathcal{H}^{\prime \prime \prime}\right) \gamma_{3}\right]-\frac{3 \Omega_{m}}{2 \mathcal{H}^{\prime}}
\end{aligned}
$$

Further derivatives give $\gamma_{1}, \gamma_{2}$ and $\gamma_{3}$. The later two particularly involve extremely complicated expressions that cannot be written down here.

When constraining 6 derivatives of the Hubble parameter from data, it is expected that the constraints will be poor. The top two panels of Fig 5 show the constraints on the initial parameters. For reasons discussed in section II, we will now linearize the relations for the modified gravity parameters about the mean likelihood values of the inital parameters. In this way we illustrate a space of possible solutions. (There would be a number of isolated solutions not represented in these results, but because these are isolated solutions they will also be suppressed in the full posterior. Better data should reduce the number of such solutions.)

\section{Linearization}

Linearizing the previous equations about the mean values of the the derivatives of $\mathcal{H}$ and $\Omega_{m}$ one finds

$$
\begin{aligned}
\alpha=- & 4.52918+14.6151 \mathcal{H}^{\prime}-3.28575 \mathcal{H}^{\prime \prime}+-0.200232 \mathcal{H}^{\prime \prime \prime} \\
& \quad-0.006872 \mathcal{H}^{\prime \prime \prime \prime}+0.0004706 \mathcal{H}^{\prime \prime \prime \prime \prime}+8.5558 \times 10^{-6} \mathcal{H}^{\prime \prime \prime \prime \prime \prime \prime}+18.3942 \Omega_{m} \\
\beta=- & 0.42128+4.91222 \mathcal{H}^{\prime}-1.71918 \mathcal{H}^{\prime \prime}+0.1071 \mathcal{H}^{\prime \prime \prime} \\
& -0.003735 \mathcal{H}^{\prime \prime \prime \prime}+0.000257 \mathcal{H}^{\prime \prime \prime \prime \prime}+4.6535 \times 10^{-6} \mathcal{H}^{\prime \prime \prime \prime \prime \prime \prime}+5.39085 \Omega_{m}
\end{aligned}
$$



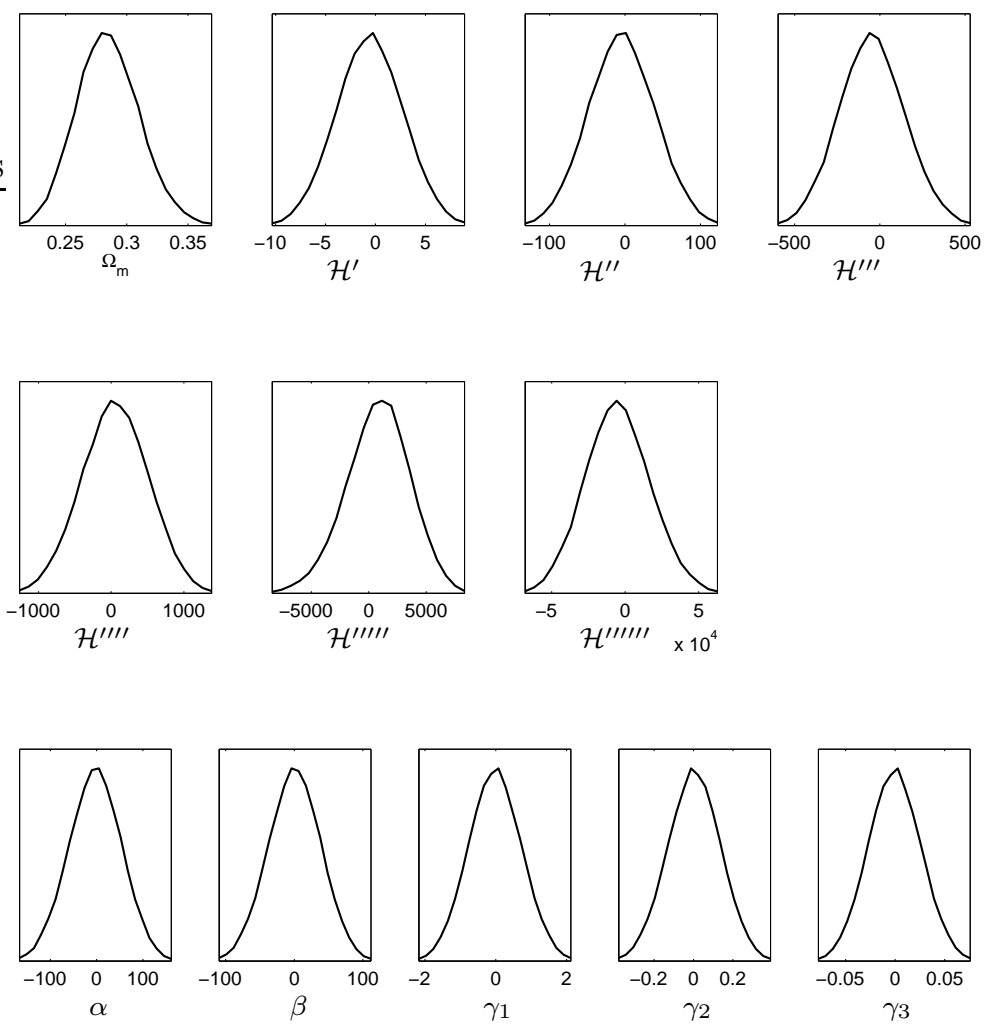

FIG. 5: Same as Fig 1, for $f\left(R, R_{\mathrm{GB}}^{2}\right)$ gravity in the metric formalism.

$$
\begin{array}{rl}
\gamma_{1}=- & 0.008276-0.05569 \mathcal{H}^{\prime}+0.03140 \mathcal{H}^{\prime \prime}-0.002392 \mathcal{H}^{\prime \prime \prime} \\
& +0.00004811 \mathcal{H}^{\prime \prime \prime \prime}-6.2764 \times 10^{-6} \mathcal{H}^{\prime \prime \prime \prime \prime}-1.03197 \times 10^{-7} \mathcal{H}^{\prime \prime \prime \prime \prime \prime \prime ~}-0.0705 \Omega_{m} \\
\gamma_{2}=0 & 0006553+0.007342 \mathcal{H}^{\prime}-0.005405 \mathcal{H}^{\prime \prime}+0.000428 \mathcal{H}^{\prime \prime \prime}-6.2403 \times 10^{-6} \mathcal{H}^{\prime \prime \prime \prime \prime} \\
& +1.2073 \times 10^{-6} \mathcal{H}^{\prime \prime \prime \prime \prime \prime}+1.9076 \times 10^{-8} \mathcal{H}^{\prime \prime \prime \prime \prime \prime \prime}+0.01237 \Omega_{m} \\
\gamma_{3}=0 & 0.0003048-0.000411 \mathcal{H}^{\prime}+0.0009694 \mathcal{H}^{\prime \prime}-0.00008026 \mathcal{H}^{\prime \prime \prime}+6.99103 \times 10^{-7} \mathcal{H}^{\prime \prime \prime \prime \prime} \\
& -2.5368 \times 10^{-7} \mathcal{H}^{\prime \prime \prime \prime \prime \prime}-3.8354 \times 10^{-9} \mathcal{H}^{\prime \prime \prime \prime \prime \prime \prime}-0.001939 \Omega_{m}
\end{array}
$$

These relations together with the MCMC chains result in the constraints shown in the bottom panel of Fig 5. The parameters are all consistent with zero, the significance is in the order of the constraints.

We have checked using the full equations that the $\gamma$ 's are in fact well constrained to be close to zero as indicated by the plot. This is because the relations contain combinations of the derivatives of $\mathcal{H}$ in their denominators. Hence this is a consequence of the equations of 
motion. This result is interesting. Again the $\mathcal{H}^{\prime \prime} \mathrm{s}=0$ is a singular point for the $\gamma$ 's (as well as for $\alpha$ and $\beta$ the relations for which contain a division by $\mathcal{H}^{\prime}$ ). Again there is a solution allowed by data that is a singular point in this theory, but overall (ie. for most allowed combinations of the derivatives of $H$ ) the parameters of the theory are well behaved, and the distributions shown encompass the main range of values for these parameters that are acceptable as per current data.

\section{DISCUSSION AND CONCLUSIONS}

$f(R)$ and $f\left(R, R_{\mathrm{GB}}^{2}\right)$ models, by definition, introduce higher derivatives of the expansion rate into the equations of motion. This automatically requires data which can allow for precise measurements of derivatives of the Hubble parameter. This severe demand on observations arises because currently we have no theory and no symmetry which can exactly predict the form of $f(R)$. This problem is evidently similar to the task of finding the form for the potential of a quintessence-like field, or more generally to finding a dynamical alternative to a cosmological constant. On the other hand, we should not feel so complacent as to accept a tiny cosmological constant as the solution to the dark energy problem without giving due consideration to the daunting dynamical alternatives in the gravity sector. In order to get rid of the possibilities discussed in this paper of modifying gravity, one may introduce the axiom that only a linear combination of Lovelock terms can enter into the Lagrangian for gravity, although this axiom does not follow from any symmetry consideration. However, this would still not be enough to solve the cosmological constant problem, namely the zeroth Lovelock term, the cosmological constant that we would predict from QFT is far too large compared to the one needed by observations. $f(R)$ models, or more generally $f\left(R, R_{\mathrm{GB}}^{2}\right)$ theories, thus remain a relevant consideration.

We have used distance measurements from CMB, BAO and SNe Ia to place preliminary bounds on the parameters of $f(R)$ models. $f(R)$ was Taylor expanded about today, keeping terms upto second order in $R$. Equations of motion were found in both the metric and Palatini formalisms. These allow us to solve for the coefficients of the Taylor expansion of $f(R)$. The equations of motion are non-linear, and we study the main space of possible solutions. The solutions are interesting in that they reflect the order of magnitude of the coefficients allowed by data. Under the metric formalism we find that $\partial f /\left.\partial R\right|_{0}$ is small 
and negative over a substantial part of the allowed region, in which case in order for these models to have a consistent GR-like evolution at early times(see [23]), this quantity must have changed sign some time in the past.

We have also analyzed a more general class of theories, $f\left(R, R_{\mathrm{GB}}^{2}\right)$. In order to modify gravity, without introducing spurious degrees of freedom (such as extra ghost-like spin-2 particles), one needs to use Lovelock scalars $[48,60]$. In four dimensions the only such terms which give a non-zero contribution are a constant, $R$, and $R_{\mathrm{GB}}^{2}$. These models are more general and the constraints are weaker. However, as for the $f(R)$ theories, we should make a serious attempt to shrink the allowed parameter space for such theories in different ways (using data and theoretical considerations).

In addition to the analysis presented here, at the moment, other considerations (convergence to GR-like evolution at early times, instabilities) are still the most serious issues that these theories need to survive. For example, it is known that some models for $f(R)$ and $f\left(R, R_{\mathrm{GB}}^{2}\right)$ do not have a FRW background consistent with GR at early times [20, 61]. Furthermore at high-redshift GR-like models may lead to unstable behaviour in the evolution of perturbations, because of the existence of either a tachionic mode or a mode with imaginary speed of propagation $[23,35,62]$. Such considerations will further constrain the parameter space of the models considered here. In this paper we have also not considered solar system type constraints, for reasons discussed in the Introduction.

To conclude, we have used current data to place constraints on the first and second derivatives of general functions of the Ricci and the Gauss-Bonnet scalars, under different formalisms. These derivatives are important quantities for theoretical considerations relating to ghosts and instabilities. Next generation dark energy surveys [63-65] can measure the cosmic expansion history much more precisely; this would dramatically shrink the presently allowed parameter space of the modified gravity models considered here. 


\section{ACKNOWLEDGEMENTS}

We thank Andrew Liddle and Mark Hindmarsh for helpful comments. ADF and PM are supported by STFC, UK. YW is supported in part by NSF CAREER grants AST-0094335.

[1] A. G. Riess, et al., Astron. J., 116, 1009 (1998)

[2] S. Perlmutter, et al., ApJ, 517, 565(1999)

[3] R. H. Dicke, Phys. Rev. 125 (1962) 2163.

[4] S. M. Carroll, A. De Felice and M. Trodden, Phys. Rev. D 71 (2005) 023525 [arXiv:astro$\mathrm{ph} / 0408081]$.

[5] L. Perivolaropoulos, Phys. Rev. D 67 (2003) 123516 [arXiv:hep-ph/0301237].

[6] V. Faraoni, Int. J. Mod. Phys. D 11 (2002) 471 [arXiv:astro-ph/0110067].

[7] A. Riazuelo and J. P. Uzan, Phys. Rev. D 66 (2002) 023525 [arXiv:astro-ph/0107386].

[8] J. P. Uzan, Phys. Rev. D 59 (1999) 123510 [arXiv:gr-qc/9903004].

[9] F. Perrotta and C. Baccigalupi, Phys. Rev. D 65 (2002) 123505 [arXiv:astro-ph/0201335].

[10] V. Pettorino, C. Baccigalupi and G. Mangano, JCAP 0501 (2005) 014 [arXiv:astro$\mathrm{ph} / 0412334]$.

[11] X. l. Chen and M. Kamionkowski, Phys. Rev. D 60 (1999) 104036 [arXiv:astro-ph/9905368].

[12] D. F. Torres, Phys. Rev. D 66 (2002) 043522 [arXiv:astro-ph/0204504].

[13] C. Santos and R. Gregory, Annals Phys. 258 (1997) 111 [arXiv:gr-qc/9611065].

[14] A. De Felice and M. Trodden, Phys. Rev. D 72 (2005) 043512 [arXiv:hep-ph/0412020].

[15] A. De Felice, G. Mangano, P. D. Serpico and M. Trodden, Phys. Rev. D 74, 103005 (2006) [arXiv:astro-ph/0510359].

[16] S. M. Carroll, V. Duvvuri, M. Trodden and M. S. Turner, Phys. Rev. D 70, 043528 (2004) [arXiv:astro-ph/0306438].

[17] S. Capozziello, S. Carloni and A. Troisi, arXiv:astro-ph/0303041.

[18] S. Tsujikawa, arXiv:0705.1032 [astro-ph].

[19] S. Fay, R. Tavakol and S. Tsujikawa, Phys. Rev. D 75, 063509 (2007) [arXiv:astro-ph/0701479].

[20] L. Amendola, R. Gannouji, D. Polarski and S. Tsujikawa, Phys. Rev. D 75, 083504 (2007) [arXiv:gr-qc/0612180]. 
[21] S. A. Appleby and R. A. Battye, arXiv:0705.3199 [astro-ph].

[22] M. S. Movahed, S. Baghram and S. Rahvar, arXiv:0705.0889 [astro-ph].

[23] I. Sawicki and W. Hu, arXiv:astro-ph/0702278.

[24] R. Bean, D. Bernat, L. Pogosian, A. Silvestri and M. Trodden, Phys. Rev. D 75, 064020 (2007) [arXiv:astro-ph/0611321].

[25] V. Faraoni, Phys. Rev. D 72, 124005 (2005) [arXiv:gr-qc/0511094].

[26] Y. S. Song, W. Hu and I. Sawicki, Phys. Rev. D 75, 044004 (2007) [arXiv:astro-ph/0610532].

[27] L. Randall and R. Sundrum, Phys. Rev. Lett. 83, 3370 (1999) [arXiv:hep-ph/9905221].

[28] G. R. Dvali, G. Gabadadze and M. Porrati, Phys. Lett. B 485, 208 (2000) [arXiv:hepth/0005016].

[29] C. Deffayet, G. R. Dvali, G. Gabadadze and A. I. Vainshtein, Phys. Rev. D 65, 044026 (2002) [arXiv:hep-th/0106001].

[30] C. Deffayet, G. R. Dvali and G. Gabadadze, Phys. Rev. D 65, 044023 (2002) [arXiv:astro$\mathrm{ph} / 0105068]$.

[31] K. Koyama, Phys. Rev. D 72, 123511 (2005) [arXiv:hep-th/0503191].

[32] S. M. Carroll, A. De Felice, V. Duvvuri, D. A. Easson, M. Trodden and M. S. Turner, Phys. Rev. D 71, 063513 (2005) [arXiv:astro-ph/0410031].

[33] O. Mena, J. Santiago and J. Weller, arXiv:astro-ph/0510453.

[34] I. Navarro and K. Van Acoleyen, Phys. Lett. B 622, 1 (2005) [arXiv:gr-qc/0506096].

[35] A. De Felice, M. Hindmarsh and M. Trodden, JCAP 0608, 005 (2006) [arXiv:astro$\mathrm{ph} / 0604154]$.

[36] G. Calcagni, B. de Carlos and A. De Felice, Nucl. Phys. B 752, 404 (2006) [arXiv:hepth/0604201].

[37] M. Sahlen, A. R. Liddle and D. Parkinson, Phys. Rev. D 75, 023502 (2007) [arXiv:astro$\mathrm{ph} / 0610812]$.

[38] W. Hu and I. Sawicki, arXiv:0705.1158 [astro-ph].

[39] T. Chiba, T. L. Smith and A. L. Erickcek, arXiv:astro-ph/0611867.

[40] A. Rajaraman, arXiv:astro-ph/0311160.

[41] T. Multamaki and I. Vilja, Phys. Rev. D 74, 064022 (2006) [arXiv:astro-ph/0606373].

[42] V. Faraoni, Phys. Rev. D 74, 023529 (2006) [arXiv:gr-qc/0607016].

[43] T. Faulkner, M. Tegmark, E. F. Bunn and Y. Mao, arXiv:astro-ph/0612569. 
[44] G. Allemandi, M. Francaviglia, M. L. Ruggiero and A. Tartaglia, Gen. Rel. Grav. 37, 1891 (2005) [arXiv:gr-qc/0506123].

[45] D. F. Mota and J. D. Barrow, Mon. Not. Roy. Astron. Soc. 349, 291 (2004) [arXiv:astro$\mathrm{ph} / 0309273]$.

[46] I. Navarro and K. Van Acoleyen, JCAP 0702, 022 (2007) [arXiv:gr-qc/0611127].

[47] A. D. Dolgov and M. Kawasaki, Phys. Lett. B 573, 1 (2003) [arXiv:astro-ph/0307285].

[48] A. Nunez and S. Solganik, Phys. Lett. B 608, 189 (2005) [arXiv:hep-th/0411102].

[49] T. Chiba, JCAP 0503, 008 (2005) [arXiv:gr-qc/0502070].

[50] A. G. Riess et al., arXiv:astro-ph/0611572.

[51] Y. Wang and P. Mukherjee, arXiv:astro-ph/0703780.

[52] D. N. Spergel et al., arXiv:astro-ph/0603449.

[53] D. J. Eisenstein et al. [SDSS Collaboration], Astrophys. J. 633, 560 (2005) [arXiv:astro$\mathrm{ph} / 0501171]$.

[54] Y. Wang and P. Mukherjee, Astrophys. J. 606, 654 (2004) [arXiv:astro-ph/0312192].

[55] Y. Wang and M. Tegmark, Phys. Rev. Lett., 92, 241302 (2004) [arXiv:astro-ph/0403292]

[56] Y. Wang and M. Tegmark, Phys. Rev. D 71, 103513 (2005) [arXiv:astro-ph/0501351].

[57] T. P. Sotiriou, Phys. Rev. D 73, 063515 (2006) [arXiv:gr-qc/0509029].

[58] G. J. Olmo and W. Komp, arXiv:gr-qc/0403092.

[59] S. Capozziello, V. F. Cardone and M. Francaviglia, Gen. Rel. Grav. 38, 711 (2006) [arXiv:astro-ph/0410135].

[60] A. Hindawi, B. A. Ovrut and D. Waldram, Phys. Rev. D 53, 5583 (1996) [arXiv:hepth/9509142].

[61] A. De Felice and M. Hindmarsh, arXiv:0705.3375 [astro-ph].

[62] Baojiu Li, John D. Barrow, David F. Mota, arXiv:0705.3795 [gr-qc]

[63] A. Albrecht; G. Bernstein; R. Cahn; W. L. Freedman; J. Hewitt; W. Hu; J. Huth; M. Kamionkowski; E.W. Kolb; L. Knox; J.C. Mather; S. Staggs; N.B. Suntzeff, Report of the Dark Energy Task Force [arXiv:astro-ph/0609591]

[64] See for example, http://www.astro.ubc.ca/LMT/alpaca/; http://www.lsst.org/; http://www.as.utexas.edu/hetdex/. [63] contains a more complete list of future dark energy experiments.

[65] Y. Wang, et al., BAAS, v36, n5, 1560 (2004); A. Crotts, et al. (2005) [arXiv:astro-ph/0507043]; 
E. Cheng; Y. Wang; et al., Proc. of SPIE, Vol. 6265, 626529 (2006); http://jedi.nhn.ou.edu/

\section{APPENDIX A: INVERTING $H(z)$ 'S TO THE DERIVATIVES OF $\mathcal{H}$}

Consider the vector $h_{i}=\mathcal{H}_{i}-1=H\left(z_{i}\right) / H_{0}-1$. If the Hubble parameter is measured in $n$ redshift bins, one can solve for $n$ of its (present day) derivatives by assuming a truncated Taylor expansion for each of $h_{i}$ about today upto order $n$, which gives

$$
\left.h_{i} \approx \sum_{j=1}^{n} \frac{d^{j} \mathcal{H}}{d z^{j}}\right|_{z=0} \frac{z_{i}^{j}}{j !} .
$$

This is a linear equation, $h_{i}=\left.A_{i j} \frac{d^{j} \mathcal{H}}{d z^{j}}\right|_{0}$, where $A_{i j}=z_{i}^{j} / j$ !, which can be inverted to find derivatives of $\mathcal{H}$ (today). We then change variables from $z$ to $N$, where $N=-\ln (1+z)$. One has

$$
\frac{d^{i} \mathcal{H}}{d z^{i}}=(-1)^{i}\left[e^{N} \frac{d}{d N}\right]^{i} \mathcal{H}
$$

so that

$$
\begin{aligned}
& \left.\frac{d \mathcal{H}}{d z}\right|_{0}=-\mathcal{H}_{0}^{\prime} \\
& \left.\frac{d^{2} \mathcal{H}}{d z^{2}}\right|_{0}=\mathcal{H}_{0}^{\prime \prime}+\mathcal{H}_{0}^{\prime} \\
& \left.\frac{d^{3} \mathcal{H}}{d z^{3}}\right|_{0}=-\left(\mathcal{H}_{0}^{(3)}+3 \mathcal{H}_{0}^{\prime \prime}+2 \mathcal{H}_{0}^{\prime}\right) \\
& \left.\frac{d^{4} \mathcal{H}}{d z^{4}}\right|_{0}=\mathcal{H}_{0}^{(4)}+6 \mathcal{H}_{0}^{(3)}+11 \mathcal{H}_{0}^{\prime \prime}+6 \mathcal{H}_{0}^{\prime} \\
& \left.\frac{d^{5} \mathcal{H}}{d z^{5}}\right|_{0}=-\left(\mathcal{H}_{0}^{(5)}+10 \mathcal{H}_{0}^{(4)}+35 \mathcal{H}_{0}^{(3)}+50 \mathcal{H}_{0}^{\prime \prime}+24 \mathcal{H}_{0}^{\prime}\right) \\
& \left.\frac{d^{6} \mathcal{H}}{d z^{6}}\right|_{0}=\mathcal{H}_{0}^{(6)}+15 \mathcal{H}_{0}^{(5)}+85 \mathcal{H}_{0}^{(4)}+225 \mathcal{H}_{0}^{(3)}+274 \mathcal{H}_{0}^{\prime \prime}+120 \mathcal{H}_{0}^{\prime} .
\end{aligned}
$$

In fact it can be shown that

$$
\left.\frac{d^{k} \mathcal{H}}{d z^{k}}\right|_{0}=(-1)^{k} \sum_{j=1}^{k} a_{k j} \mathcal{H}_{0}^{(j)}
$$

with

$$
\begin{aligned}
& a_{k 1}=(k-1) ! \\
& a_{k j}=(k-1) a_{k-1, j}+a_{k-1, j-1}, \quad 1<j<k \\
& a_{k k}=1
\end{aligned}
$$


This then specifies the whole series of polynomials.

Writing the linear relation as

$$
\left.\frac{d^{j} \mathcal{H}}{d z^{j}}\right|_{0}=B_{j k} \mathcal{H}_{0}^{(k)}
$$

we have

$$
h_{i}=A_{i j} B_{j k} \mathcal{H}_{0}^{(k)} \equiv M_{i k}^{-1} \mathcal{H}_{0}^{(k)}
$$

or

$$
\mathcal{H}_{0}^{(k)}=M_{k j} h_{j}
$$

For two bins case $\left(z_{1}=0.7\right.$, and $z_{2}=1.4$ for current data) we get

$$
M=\left(\begin{array}{ll}
-2.85714 & 0.714286 \\
-1.22449 & 1.32653
\end{array}\right)
$$

For three bins $\left(z_{1}=0.4667, z_{2}=0.9333\right.$, and $\left.z_{3}=1.4\right)$ then we get

$$
M=\left(\begin{array}{ccc}
-6.4286 & 3.2143 & -0.71429 \\
-16.531 & 15.153 & -3.8775 \\
32.930 & -22.369 & 3.2216
\end{array}\right)
$$

for 4 bins equally spaced out to $z=1.4$, we get

$$
M=\left(\begin{array}{cccc}
-11.429 & 8.5714 & -3.8095 & 0.71428 \\
-59.320 & 68.980 & -34.286 & 6.7687 \\
-9.0962 & 55.802 & -52.789 & 13.251 \\
509.11 & -745.18 & 450.18 & -91.607
\end{array}\right)
$$

for 5 bins, we have

$$
M=\left(\begin{array}{cccccc}
-17.857 & 17.857 & -11.905 & 4.4647 & -0.71429 \\
-145.83 & 209.61 & -153.91 & 60.374 & -9.9150 \\
-335.37 & 679.30 & -630.53 & 276.88 & -48.546 \\
1445.8 & -2258.6 & 1643.0 & -562.54 & 79.241 \\
2094.7 & -6287.7 & 7809.3 & -4285.9 & 838.55
\end{array}\right)
$$


and, finally, for 6 bins, we have

$$
M=\left(\begin{array}{rrrrrrr}
-19.3 & 16.109 & -7.193 & 0.03758 & 1.271 & -0.3546 \\
-179.35 & 218.78 & -108.04 & 0.67106 & 20.4867 & -5.823 \\
-500.66 & 833.8 & -523.69 & 4.9750 & 118.16 & -35.142 \\
1837.10 & -2403.5 & 1168.7 & 4.745 & -149.15 & 32.4457 \\
4187.9 & -9101.2 & 7205.2 & -112.35 & -2310.5 & 732.79 \\
-54953 & 91546 & -59415 & 191.52 & 14897 & -4190.4
\end{array}\right)
$$

With the present day derivatives of the Hubble parameter thus derived from model independent measurements of the Hubble parameter in linear redshift bins from cosmological data, we can then obtain constraints on parameters of modified gravity models, using appropriate equations of motion. This is done in sections II,III and IV of this paper.

\section{APPENDIX B: APPENDIX B: SOLUTIONS FOR THE PALATINI FORMALISM}

We could find the following distinct solutions of the equations $F_{i}\left(\bar{p}_{j}, \bar{q}_{k}\right)=0$ for $\alpha, \beta$, and $\gamma$, about the mean values for $\bar{q}_{k}=\left(\Omega_{m} \cdot \mathcal{H}^{\prime}, \mathcal{H}^{\prime \prime}\right):[-0.857943,-0.428971$, 0.14299], [-1.46637, -0.422284, -0.140728], [0.399627, 0.824305, 0.852729], [3.78266, 0.748323, -0.00792803], [0.222102, 0.00488155, 4.12207e-05], [-0.739164, -0.00165984, 0.0000280094], [-0.866737, -0.00527848, 0.000315044], [0.252698, -0.00306054, 0.0000152404], [-0.29676, 0.00333869, 0.0000390608], [-0.811148, -0.00218394, 0.0000614933], [-0.410838, -0.0124848, $-0.0000849403],[-0.132685,-0.00276524,0.0000205499]$, and [0.00810481, -0.00504213 , $0.00002751]$.

For the solution $[0.222102,0.00488155,4.12207 \mathrm{e}-05]$, the $A^{-1} B$ of equation (34) is

$$
A^{-1} B=\left(\begin{array}{ccc}
8.7943 & -0.0077692 & 0.000655508 \\
11.162 & -0.015052 & 0.0012888 \\
0.18687 & -0.00025239 & 0.000021581
\end{array}\right) \text {. }
$$

